\title{
Have 100 years of talking therapies taught psychiatrists how to communicate?
}

\author{
Jon Wilson
}

COMMENTARY ON... COCHRANE CORNER ${ }^{\dagger}$

\begin{abstract}
SUMMARY
Formal communication skills training is the norm at UK medical schools, and evidence is mounting that it is effective in a number of domains, including patient satisfaction and shared decision-making, leading to improved treatment adherence. However, applying these models in mental health settings is challenging, despite the fact that positive therapeutic relationships and an ability to actively manage consultations are the cornerstone of psychiatric practice. Communication skills training is still too often regarded as better suited to physical health consultations than more complex psychiatric interviews. Here, it is considered why this might be, with reference to the one eligible randomised controlled trial identified in this systematic literature review.
\end{abstract}

\section{DECLARATION OF INTEREST}

J.W. has previously worked with Professor Papageorgiou at the University of East Anglia and has authored chapters in books that she has coedited.

\section{KEYWORDS}

Randomised control trial; communication; schizophrenia; severe mental illness; communication skills training; community; Cochrane review.

It seems staggering that 100 years after Freud and colleagues first began to highlight the importance of talking therapies there is, according to the review featured in this month's Cochrane Corner (Papageorgiou 2017), only one randomised controlled trial (RCT) specifically addressing how the manner in which consultations are conducted affects any form of clinical outcome in a patient group with severe mental illness. There are perhaps many reasons why this might be the case. However, given that communication skills are embedded in the curriculum at most UK medical schools, and taught extensively around the world, this lack of empirical evidence seems a clear gap.

\section{What is communication skills training?}

Formal communication skills training is a relatively new phenomenon on the scale that it is currently taught, which may in part explain why there a paucity of evidence about it. In essence, it aims to teach skills that facilitate a better relationship between the doctor and patient, even in challenging situations. In turn, this aims to ensure that patients are fully informed and engaged in their care and ultimately it should lead to improved satisfaction and clinical outcomes. Disciplines such as primary care have long known this and have actively sought to mandate its inclusion in their training programmes. Psychiatry, however, has unfortunately long thought of itself as the expert communication discipline and therefore been more sceptical of what is often seen as a formulaic approach to the sacred psychiatric interview. It is possible, though, that we have been deceiving ourselves.

\section{Does psychiatry need it?}

There is no doubt that the communication skills necessary to practise psychiatry are a step up in complexity compared with those needed in physical medicine. There are a variety of communication skills models or conducting consultations, the dominant current UK model being the CalgaryCambridge model (Silverman 2013). Others, such as the three-function approach (Bird 1990) and the Kalamazoo Consensus Statement's essential elements (Makoul 2001), follow similar theoretical concepts and all have the aim of dividing a consultation into a series of tasks to be actively managed. Although this division into tasks or small sections seems artificial, it provides the clinician with a framework to ensure that information is gathered systematically, sensitively and optimally, that knowledge is transferred effectively and that treatment decisions are collaborative. The tasks themselves are each broken down into a number of discrete skills, such as active listening, chunking and checking, and recognising, acknowledging and validating the patient's
ROUND THE CORNER
Jon Wilson is a consultant psychiatrist and research director within Norfolk and Suffolk NHS Foundation Trust, and a clinical senior lecturer in Norwich Medical School at the University of East Anglia, UK. He specialises in youth mental health but has a clinical background in recovery and early intervention in psychosis and is a medical psychotherapist.

Correspondence Dr Jon Wilson, 80 St Stephens Road, Norwich NR1 3RE, UK. Email: jon.wilson@nsft.nhs.uk

\section{Copyright and usage} (C) The Royal College of Psychiatrists 2018

${ }^{\dagger}$ See p. 220, this issue. 


\begin{tabular}{|ll|}
\hline Tasks & \multicolumn{1}{c|}{ Skills } \\
\hline Establishing initial rapport & Uses open questions \\
& Active listening without interruption \\
Negotiating an agenda & Explicitly takes both the patient's and the clinician's views and needs into account \\
& Actively determines the patient's ideas, concerns and expectations (ICE) \\
Gathering information & Repeatedly moves from open to closed questions, signposting in between themes \\
& Clarifies statements \\
& Screens for further information ('Is there anything else?') \\
& Summarises at the end of each logical sequence, and signposts to the next topic \\
Providing structure & Uses a logical interview structure with discrete sections \\
& Chunks information given and repeatedly checks understanding \\
Building rapport & Verbally and non-verbally encourages responses \\
& Listens attentively, picks up on the patient's cues \\
& Validates the patient's feelings appropriately \\
Involving the patient & Assesses the patient's baseline knowledge and views \\
& Shares thinking \\
& Explains rationale and options clearly \\
\hline
\end{tabular}

Sources: Coll et al (2012); Silverman et al (2013).

ideas, concerns and expectations (Table 1). Once learnt in context and skilfully applied, these skills can add to the artistry of a consultation, thereby allowing the patient's voice to be heard and leading to genuine shared decision-making. Regrettably, too many 'experienced' psychiatrists still assume that they have learnt these skills over the years, without ever subjecting themselves to outside scrutiny or feedback. In reality, the psychiatric patient's experience is generally not of robust collaborative decision-making and, unfortunately, this is frequently rationalised away as somehow the fault of the 'recipient' rather than the 'director' of the consultation. Too often, the quality of the interview is not even considered.

\section{Might trainees be putting their consultants to shame?}

If you have any doubt that such skills are effective, then observe a top candidate performing the Clinical Assessment of Skills and Competencies (CASC) element of the Royal College of Psychiatrists' membership examinations as they skilfully direct a consultation with clear goals and numerous opportunities for the (simulated) patient to express their point of view, and strive to achieve a shared decision, often within a complex and challenging encounter. These psychiatric trainees have been taught such approaches to actively managing consultations, observing the consultation at a meta-level and preempting its direction through use of empathy and ensuring that the patient's concerns are continually addressed. By contrast, many senior clinicians have not had opportunities to put themselves through the process of improving such skills through any method other than having spoken to patients over many years with no feedback, and they have widely varying abilities to actively manage a therapeutic relationship. Too often these individuals are critical of the modern concept of teaching communication, citing a dumbing down of complex issues and the creation of wooden or formulaic approaches to patients. Taught well, nothing could be further from reality.

\section{Why does psychiatry resist?}

Communication skills can be learnt from books, online or through didactic teaching (Box 1). However, the gold standard of communication skills

BOX 1 Further reading/online resources

'A three-talk model for shared decision making: multistage consultation process' (Elwyn 2017)

A simple and effective model described in the BMJ focusing on shared decision-making in medicine

Clinical Communication in Medicine (Brown 2016) A detailed book bringing together the theories and evidence base underpinning effective healthcare communication

Communication Skills in Mental Health Care: An Introduction (Coll 2012)

A practical book outlining the essential features of the Calgary-Cambridge model of communication skills, tailored and applied to various psychiatric diagnoses and settings

Skills for Communicating with Patients (Silverman 2013)

A usable but definitive book outlining the theory and use of the Calgary-Cambridge model across medicinal disciplines

UK Council for Clinical Communication (www.ukccc. org.uk)

An excellent online resource offering practical explanations of the essential elements of communication skills; includes video material 
training involves using simulated patients within a peer learning setting. This exposes the individual, but allows them to be observed and receive feedback or strategies for improvement and to have direct 'patient' feedback. This approach is increasingly popular in the physical medical disciplines, yet senior psychiatrists seem to continue to stubbornly resist it. Arguably, this may be because of the complexity of psychiatric presentations and the need to balance both the experience and the skills that are necessary to respond to and direct the bewildering number of interpersonal interactions that occur during a consultation. Indeed, the vast majority of research in communication skills training has been undertaken in physical healthcare settings. There is increasing and extensive evidence in physical healthcare that such approaches are acceptable and beneficial. Brown et al (2016) comprehensively set out the evidence underpinning such approaches in a variety of settings. Covering all areas of medicine, their book even includes a few chapters on clinical communication in mental healthcare, outlining the unique aspects of this area of medical practice.

In psychiatric practice the complexity of the patients' presentations, backgrounds, transferences and countertransferences and of the settings themselves makes standardisation of these clinical encounters endlessly challenging. However, even within psychiatry there are clear themes, approaches, attitudes and skills that can be reliably utilised. The basic skills have been collated by Coll et al (2012), in a book that includes examples of how to approach a variety of psychiatric encounters. In it, Papageorgiou and colleagues have developed these skills and tailored them to psychiatric practice within an undergraduate curriculum, using the tried and tested method of simulated patient encounters and role-play. This extension of core skills to complex and unpredictable situations allows the worlds of psychotherapy and empirical psychiatry to meet in the application of a set of skills still clearly linked to the Calgary-Cambridge model of communication. It is disappointing to read in this Cochrane review that, despite the evidence that such skills lead to improved outcomes in general medicine (Brown 2016), the evidence base is yet to inform a robust argument for such communication training to be uniformly accepted at all levels of psychiatric seniority.

\section{The Cochrane review}

In their Cochrane review, Papageorgiou and colleagues seek to identify the existing evidence for the use of such skills in psychiatric practice using RCTs as the gold standard for definitive evidence (Papageorgiou 2017). It comes as a great surprise that they were able to identify just one reasonably robust trial, a pilot RCT. This is possibly because of their tight, but arguably necessary, criteria of ensuring that only trials investigating severe mental illness were included. Presumably this was because they wanted to explore how credible the use of such techniques is in routine psychiatric practice, rather than in general practice with patients who have comparatively minor psychological difficulties. The paucity of RCTs in this area could arguably reflect the difficulties in conceptualising and conducting sophisticated psychiatric research. However, the included trial followed relatively tried and tested methodologies, thereby demonstrating that such research was at least possible. It was clearly impossible to conduct any meta-analysis on the single trial identified by their systematic literature search.

They are explicit about the types of trial that they would have included, namely RCTs involving mental health professionals and patients with severe mental illness (defined as schizophrenia and schizophreniform disorders, or some severe mood disorders involving schizophrenia-related illnesses) in which the professionals received some form of communication skills training compared with standard or no training.

\section{The results}

The authors provide a clear and honest appraisal of the published and unpublished data from the single eligible trial (McCabe 2016). The clinicians who participated in the trial were higher psychiatry trainees who were allocated using a cluster randomised methodology (i.e. allocation was based on healthcare facility) to avoid contamination. The clinicians ( $n=21)$ were either trained in communication skills over four sessions each lasting $4 \mathrm{~h}$ or received no specific training. The patients $(n=97)$ all met ICD-10 diagnostic criteria for schizophrenia or schizoaffective disorder and were from an urban (East London, UK) setting. Outcome measures included positive and negative symptoms, patient satisfaction and therapeutic relationship, assessed using validated questionnaires - the Positive and Negative Syndrome Scale (PANSS), Client Satisfaction Questionnaire (CSQ-8) and the Scale To Assess Therapeutic Relationship (STAR).

The findings of the review team are clear and speak for themselves. They indicate low-quality evidence that:

'patient satisfaction with treatment as reported by the patients did not differ between the intervention and control groups at medium-term follow-up, although there was a modest improvement in patients' satisfaction with the therapeutic relationship in the intervention group as compared to control. Equally, there was no significant difference in the mental state scores of the patients when comparing the CST [communication skills training] group and NST [no specific 
training] group at medium-term follow-up, nor any difference in numbers leaving early'.

\section{However,}

'Five months after treatment, patients who were treated by psychiatrists who received communication training had a modest increase in satisfaction with the therapeutic relationship compared with patients treated by psychiatrists who did not receive the training. Satisfaction with treatment and mental state of the patient were similar between the two treatment groups'.

Although encouraging, this is not robust evidence either way in deciding whether resources should be diverted to the ongoing training of psychiatric skills to trainees using taught models or even to making routine teaching available to established psychiatric practitioners and other senior clinicians.

\section{Why such an absence of evidence?}

Unfortunately, this Cochrane review culminates in a familiar refrain:

'Due to the small sample size and the exploratory nature of this [...] RCT, it is difficult to draw robust conclusions on the treatment effect. More, and larger scale studies in psychiatry are needed in order to collect evidence on the effectiveness of clinical communication training on the above outcomes'.
This is regrettable since any intervention aimed specifically at improving psychiatric patients' understanding, autonomy and, ultimately, adherence to an agreed treatment plan would undoubtedly have significant clinical and financial implications for patient care. One wonders why such robust evidence does not seem to exist, despite 100 years of talking therapies.

\section{References}

Bird J, Cohen-Cole SA (1990) The three-function model of the medical interview: an educational device. Advances in Psychosomatic Medicine, 20: 65-88.

Brown J, Noble LM, Papageorgiou A, et al (eds) (2016) Clinical Communication in Medicine. John Wiley \& Sons.

Coll X, Papageorgiou A, Stanley A, et al (eds) (2012) Communication Skills in Mental Health Care: An Introduction. Radcliffe Publishing.

Elwyn G, Durand MA, Song J, et al (2017) A three-talk model for shared decision making: multistage consultation process. BMJ, 359: j4891.

Makoul G (2001) Essential elements of communication in medical encounters: The Kalamazoo Consensus Statement. Academic Medicine 76: $390-3$

McCabe R, John P, Dooley J, et al (2016) Training to enhance psychiatrist communication with patients with psychosis (TEMPO): cluster randomised controlled trial. British Journal of Psychiatry, 209: 517-24.

Papageorgiou A, Loke YK, Fromage M (2017) Communication skills training for mental health professionals working with people with severe mental illness. Cochrane Database of Systematic Reviews, 6: CD010006 (doi: 10.1002/14651858.CD010006.pub2).

Silverman J, Kurtz S, Draper J (2013) Skills for Communicating with Patients (3rd edn). CRC Press 